\title{
The Generalized Bisymmetric (Bi-Skew-Symmetric) Solutions of a Class of Matrix Equations and Its Least Squares Problem
}

\author{
Yifen Ke and Changfeng Ma \\ School of Mathematics and Computer Science, Fujian Normal University, Fuzhou 350007, China \\ Correspondence should be addressed to Changfeng Ma; macf09@139.com
}

Received 24 November 2013; Accepted 18 January 2014; Published 17 March 2014

Academic Editor: Qing-Wen Wang

Copyright (C) 2014 Y. Ke and C. Ma. This is an open access article distributed under the Creative Commons Attribution License, which permits unrestricted use, distribution, and reproduction in any medium, provided the original work is properly cited.

\begin{abstract}
The solvability conditions and the general expression of the generalized bisymmetric and bi-skew-symmetric solutions of a class of matrix equations $(A X=B, X C=D)$ are established, respectively. If the solvability conditions are not satisfied, the generalized bisymmetric and bi-skew-symmetric least squares solutions of the matrix equations are considered. In addition, two algorithms are provided to compute the generalized bisymmetric and bi-skew-symmetric least squares solutions. Numerical experiments illustrate that the results are reasonable.
\end{abstract}

\section{Introduction}

The class of matrix equations, namely, $A X=B, X C=D$, where $A, B, C$, and $D$ are given, is one of the most interesting and intensively studied classes of linear algebra. It has been investigated by many authors and a series of important and useful results has been obtained (see, e.g., [1-31]).

For example, Cecioni [1] gave a necessary and sufficient condition for the matrix equations to have a common solution and a general expression of the common solution was obtained by Rao and Mitra ([2], page 25) ; Mitra [3] obtained a common solution with the minimum possible rank and also other feasible specified rank; Chu [4] achieved new necessary and sufficient conditions for the matrix equations by using the generalized singular value decomposition. In [5], Wang and $\mathrm{Yu}$ derived the necessary and sufficient conditions and the expressions for the orthogonal solutions, the symmetric orthogonal solutions, and the skew-symmetric orthogonal solutions of the matrix equations, respectively. Khatri and Mitra [6] considered the general Hermitian and nonnegative definite solutions of the matrix equations, respectively. Dajić and Koliha [7] studied the positive solutions to the matrix equations for Hilbert space operators using generalized inverses, and a sufficient and necessary condition for its solvability and a representation of its general solutions were also established therein. Li et al. investigated the generalized reflexive and antireflexive solution of the matrix equations, in
$[8,9]$, respectively. In [10], Qiu et al. considered the unknown matrix $X$ with the constraint $P X=s X P$, where $P$ is a given Hermitian matrix satisfying $P^{2}=I$ and $s= \pm 1$.

In this paper, $R^{m \times n}, O R^{n \times n}, S R^{n \times n}$, and $S O R^{n \times n}$ denote the set of all $m \times n$ real matrices, the set of all $n \times n$ real orthogonal matrices, the set of all $n \times n$ real symmetric matrices, and the set of all $n \times n$ real symmetric orthogonal matrices, respectively. $A^{\top}$ represents the transpose of the real matrix $A$ and $\|\cdot\|$ stands for the Frobenius norm induced by the inner product. $\left(\begin{array}{ll}A & B\end{array}\right)$ denotes a row block matrix and $A \circ B$ denotes the Hadamard product produced by $A$ and $B$, namely, $A \circ B=\left(a_{i j} b_{i j}\right)$. The symbol $I_{n}$ stands for the identity matrix of order $n$. Let $A^{\dagger}$ be the Moore-Penrose generalized inverse of a matrix $A \in R^{m \times n}$, which is defined to be the unique solution $X \in R^{n \times m}$ satisfying the following four matrix equations:

$$
\begin{array}{cl}
\text { (1) } A X A=A, & \text { (2) } X A X=X, \\
\text { (3) }(A X)^{\top}=A X, & \text { (4) }(X A)^{\top}=X A .
\end{array}
$$

Furthermore, $\mathscr{L}_{\mathrm{A}}$ and $\mathscr{R}_{A}$ represent the two orthogonal projectors $\mathscr{L}_{A}=I_{n}-A^{\dagger} A$ and $\mathscr{R}_{A}=I_{m}-A A^{\dagger}$. Set $S_{n}=\left(e_{n}, e_{n-1}, \ldots, e_{1}\right)$, where $e_{i}$ denotes the $i$ th column of the identity matrix $I_{n}$. It is easy to see that $S_{n}^{\top}=S_{n}$ and $S_{n}^{\top} S_{n}=I_{n}$.

Definition 1. Let $P \in S O R^{n \times n}$; that is, $P=P^{\top}=P^{-1}$. A matrix $X \in R^{n \times n}$ is said to be a generalized bisymmetric matrix if 
$X=X^{\top}=P X P$. The set of all $n \times n$ generalized bisymmetric matrices is denoted by $G B S R^{n \times n}$.

Definition 2. Let $P \in S O R^{n \times n}$; that is, $P=P^{\top}=P^{-1}$. A matrix $X \in R^{n \times n}$ is said to be a generalized bi-skew-symmetric matrix if $X=-X^{\top}=-P X P$. The set of all $n \times n$ generalized bi-skew-symmetric matrices is denoted by GBSSR ${ }^{n \times n}$.

Without special statement, we assume that $P$ is a given symmetric orthogonal matrix in the remainder of this paper.

If $P=S_{n}$, then the generalized bisymmetric matrix reduces to the bisymmetric matrix and the generalized biskew-symmetric matrix reduces to the antisymmetric and persymmetric matrix.

In this paper, we consider the following problems.

Problem 3. Given $A, B \in R^{m \times n}, C, D \in R^{n \times l}$, find $X \in$ $G_{B S R}^{n \times n}$ such that

$$
A X=B, \quad X C=D .
$$

Problem 4. Given $A, B \in R^{m \times n}, C, D \in R^{n \times l}$, find $X \in$ $G_{B S S R}^{n \times n}$ such that

$$
A X=B, \quad X C=D .
$$

Problem 5. Given $A, B \in R^{m \times n}, C, D \in R^{n \times l}$, find $\widehat{X} \in$ $G_{B S R}^{n \times n}$ such that

$$
\|A \widehat{X}-B\|+\|\widehat{X} C-D\|=\min _{X \in G B S R^{n \times n}}\|A X-B\|+\|X C-D\| .
$$

Problem 6. Given $A, B \in R^{m \times n}, C, D \in R^{n \times l}$, find $\widehat{X} \in$ $G_{B S S R}{ }^{n \times n}$ such that

$$
\|A \widehat{X}-B\|+\|\widehat{X} C-D\|=\min _{X \in G B S S R^{n \times n}}\|A X-B\|+\|X C-D\| .
$$

If $C=D=0$, then those problems become the problems discussed in [11]. So, this paper extends the part results of [11]. In our work, the necessary and sufficient conditions for the existence of the solutions to Problems 3 and 4 are derived and their general expressions of the solutions are given by Moore-Penrose generalized inverse, respectively. If the solvability conditions are not satisfied, Problems 5 and 6 will be considered.

The remainder of this paper is arranged as follows. In Section 2, we establish the necessary and sufficient conditions and the explicit expressions of Problems 3 and 4. In Section 3, we investigate Problems 5 and 6 by virtue of the singular value decomposition (SVD) and the special decompositions of the generalized bisymmetric matrices and the generalized bi-skew-symmetric matrices. In Section 4, we give two algorithms and some examples to illustrate the efficiency of our proposed results. In Section 5, some conclusions are made.

\section{The Generalized Bisymmetric (Bi-Skew- Symmetric) Solutions of the Matrix \\ Equations $A X=B, X C=D$}

In this section, we first recall some lemmas which will be used for obtaining the necessary and sufficient conditions and the explicit expressions of Problems 3 and 4.

Lemma 7 (see [12]). Assume $P \in S O R^{n \times n}$, and let

$$
P_{1}=\frac{1}{2}\left(I_{n}+P\right), \quad P_{2}=\frac{1}{2}\left(I_{n}-P\right) .
$$

Then $P_{1}$ and $P_{2}$ are orthogonal projection matrices satisfying $P_{1}+P_{2}=I_{n}, P_{1} P_{2}=0$. Furthermore, assume $\operatorname{rank}\left(P_{1}\right)=r$. Then, $\operatorname{rank}\left(P_{2}\right)=n-r$ and there exist unit column orthogonal matrices $U_{1} \in R^{n \times r}$ and $U_{2} \in R^{n \times(n-r)}$ such that

$$
\begin{gathered}
P_{1}=U_{1} U_{1}^{\top}, \quad P_{2}=U_{2} U_{2}^{\top}, \\
P=U_{1} U_{1}^{\top}-U_{1} U_{1}^{\top}, \quad U_{1}^{\top} U_{2}=0 .
\end{gathered}
$$

From Lemma 7, we note that $U=\left(\begin{array}{ll}U_{1} & U_{2}\end{array}\right)$ is an orthogonal matrix and the symmetric orthogonal matrix $P$ can be expressed as

$$
P=U\left(\begin{array}{cc}
I_{r} & 0 \\
0 & -I_{n-r}
\end{array}\right) U^{\top} .
$$

Lemma 8 (see [11]). Assume that the spectral decomposition of $P$ is given as in (8). Then $X \in G B S R^{n \times n}$ if and only if $X$ can be expressed as

$$
X=U\left(\begin{array}{cc}
X_{11} & 0 \\
0 & X_{22}
\end{array}\right) U^{\top},
$$

where $X_{11} \in S R^{r \times r}$ and $X_{22} \in S R^{(n-r) \times(n-r)}$.

Proof. For $X \in G B S R^{n \times n}$, by Definition 1, it is easy to know that $P X=X P$. Then, we have

$$
\begin{aligned}
P_{1} X P_{1} & =\frac{I_{n}+P}{2} X \frac{I_{n}+P}{2} \\
& =\frac{1}{4}(X+P X+X P+P X P)=\frac{1}{2}(X+P X), \\
P_{2} X P_{2} & =\frac{I_{n}-P}{2} X \frac{I_{n}-P}{2}=\frac{1}{4}(X-P X-X P+P X P) \\
& =\frac{1}{2}(X-P X) .
\end{aligned}
$$

By (10) and Lemma 7, we obtain

$$
\begin{aligned}
X & =P_{1} X P_{1}+P_{2} X P_{2} \\
& =U_{1} U_{1}^{\top} X U_{1} U_{1}^{\top}+U_{2} U_{2}^{\top} X U_{2} U_{2}^{\top} .
\end{aligned}
$$

Let $X_{11}=U_{1}^{\top} X U_{1}$ and $X_{22}=U_{2}^{\top} X U_{2}$; it is easy to verify that $X_{11}=X_{11}^{\top}$ and $X_{22}=X_{22}^{\top 2}$. Furthermore, we have

$$
X=U_{1} X_{11} U_{1}^{\top}+U_{2} X_{22} U_{2}^{\top}=U\left(\begin{array}{cc}
X_{11} & 0 \\
0 & X_{22}
\end{array}\right) U^{\top} .
$$


Conversely, for any $X_{11} \in S R^{r \times r}$ and $X_{22} \in S R^{(n-r) \times(n-r)}$, it is easy to verify that $X=X^{\top}$. Using (8), we have

$$
\begin{aligned}
P X P & =U\left(\begin{array}{cc}
I_{r} & 0 \\
0 & -I_{n-r}
\end{array}\right) U^{\top} U\left(\begin{array}{cc}
X_{11} & 0 \\
0 & X_{22}
\end{array}\right) U^{\top} U\left(\begin{array}{cc}
I_{r} & 0 \\
0 & -I_{n-r}
\end{array}\right) U^{\top} \\
& =U\left(\begin{array}{cc}
X_{11} & 0 \\
0 & X_{22}
\end{array}\right) U^{\top} \\
& =X .
\end{aligned}
$$

This implies that

$$
X=U\left(\begin{array}{cc}
X_{11} & 0 \\
0 & X_{22}
\end{array}\right) U^{\top} \in G B S R^{n \times n}
$$

Lemma 9 (see [11]). Assume that the spectral decomposition of $P$ is given as in (8). Then, $X \in G B S S R^{n \times n}$ if and only if $X$ can be expressed as

$$
X=U\left(\begin{array}{cc}
0 & X_{12} \\
-X_{12}^{\top} & 0
\end{array}\right) U^{\top}
$$

where $X_{12} \in R^{r \times(n-r)}$.

Proof. For $X \in G B S S R^{n \times n}$, by Definition 2, we have

$$
\begin{aligned}
P_{1} X P_{1} & =\frac{I_{n}+P}{2} X \frac{I_{n}+P}{2} \\
& =\frac{1}{4}(X+P X+X P+P X P)=\frac{1}{4}(P X+X P), \\
P_{2} X P_{2} & =\frac{I_{n}-P}{2} X \frac{I_{n}-P}{2} \\
& =\frac{1}{4}(X-P X-X P+P X P)=-\frac{1}{4}(P X+P X) .
\end{aligned}
$$

By (16) and Lemma 7, we obtain

$$
\begin{aligned}
X & =\left(P_{1}+P_{2}\right) X\left(P_{1}+P_{2}\right) \\
& =P_{1} X P_{1}+P_{1} X P_{2}+P_{2} X P_{1}+P_{2} X P_{2} \\
& =P_{1} X P_{2}+P_{2} X P_{1} \\
& =U_{1} U_{1}^{\top} X U_{2} U_{2}^{\top}+U_{2} U_{2}^{\top} X U_{1} U_{1}^{\top} .
\end{aligned}
$$

Let $X_{12}=U_{1}^{\top} X U_{2}$ and $X_{21}=U_{2}^{\top} X U_{1}$; it is easy to verify that $X_{21}=-X_{12}^{\top}$. And we have

$$
\begin{aligned}
X & =U_{1} X_{12} U_{2}^{\top}+U_{2} X_{21} U_{1}^{\top} \\
& =U\left(\begin{array}{cc}
0 & X_{12} \\
X_{21} & 0
\end{array}\right) U^{\top}=U\left(\begin{array}{cc}
0 & X_{12} \\
-X_{12}^{\top} & 0
\end{array}\right) U^{\top} .
\end{aligned}
$$

Conversely, for any $X_{12} \in R^{r \times(n-r)}$, it is easy to verify that $X=-X^{\top}$. Using (8), we have

$$
\begin{aligned}
\text { PXP } & =U\left(\begin{array}{cc}
I_{r} & 0 \\
0 & -I_{n-r}
\end{array}\right) U^{\top} U\left(\begin{array}{cc}
0 & X_{12} \\
-X_{12}^{\top} & 0
\end{array}\right) U^{\top} U\left(\begin{array}{cc}
I_{r} & 0 \\
0 & -I_{n-r}
\end{array}\right) U^{\top} \\
& =U\left(\begin{array}{cc}
0 & -X_{12} \\
X_{12}^{\top} & 0
\end{array}\right) U^{\top} \\
& =-X .
\end{aligned}
$$

This implies that

$$
X=U\left(\begin{array}{cc}
0 & X_{12} \\
-X_{12}^{\top} & 0
\end{array}\right) U^{\top} \in G B S S R^{n \times n} .
$$

Remark 10. For Lemma 8, Wang and Yu [11] just gave the conclusion; we prove it here. The proof of Lemma 9 can be seen in [12]; for the convenience of the reader, we rewrite it.

Lemma 11 (see [13]). Suppose that $A_{1} \in R^{m \times n}, A_{3} \in R^{k \times n}$, $B_{2} \in R^{r \times s}, B_{4} \in R^{r \times l}, C_{1} \in R^{m \times r}, C_{2} \in R^{n \times s}, C_{3} \in R^{k \times r}$, $C_{4} \in R^{n \times l}$ are known and $X \in R^{n \times r}$ is unknown. Let $K=$ $A_{3} \mathscr{L}_{A_{1}}, N=\mathscr{R}_{B_{2}} B_{4}, Q_{1}=C_{3}-A_{3} A_{1}^{\dagger} C_{1}-K C_{2} B_{2}^{\dagger}, Q=$ $C_{4}-A_{1}^{\dagger} C_{1} B_{4}-\mathscr{L}_{A_{1}} C_{2} B_{2}^{\dagger} B_{4}-\mathscr{L}_{A_{1}} K^{\dagger} Q_{1} N$. Then the system of matrix equations

$$
A_{1} X=C_{1}, \quad X B_{2}=C_{2}, \quad A_{3} X=C_{3}, \quad X B_{4}=C_{4}
$$

is consistent if and only if

$$
\begin{aligned}
& K K^{\dagger} Q_{1} \mathscr{R}_{B_{2}}=Q_{1}, \quad Q \mathscr{L}_{N}=0,
\end{aligned}
$$

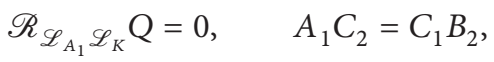

$$
\begin{aligned}
& A_{i} A_{i}^{\dagger} C_{i}=C_{i}, \quad C_{j} B_{j}^{\dagger} B_{j}=C_{j}, \quad i=1,3 ; j=2,4,
\end{aligned}
$$

in which case, the general solutions of the system can be expressed as

$$
\begin{aligned}
X= & A_{1}^{\dagger} C_{1}+\mathscr{L}_{A_{1}} C_{2} B_{2}^{\dagger}+\mathscr{L}_{A_{1}} K^{\dagger} Q_{1} \mathscr{R}_{B_{2}} \\
& +Q N^{\dagger} \mathscr{R}_{B_{2}}+\mathscr{L}_{A_{1}} \mathscr{L}_{K} Z \mathscr{R}_{N} \mathscr{R}_{B_{2}},
\end{aligned}
$$

where $Z$ is an arbitrary real matrix with compatible dimension.

Lemma 12. Given $A, B \in R^{m \times n}, C, D \in R^{n \times l}$. Let $K=C^{\top} \mathscr{L}_{A}$, $N=\mathscr{R}_{C} A^{\top}, Q_{1}=D^{\top}-C^{\top} A^{\dagger} B-K D C^{\dagger}, Q=B^{\top}-A^{\dagger} B A^{\top}-$ $\mathscr{L}_{A} D C^{\dagger} A^{\top}-\mathscr{L}_{A} K^{\dagger} Q_{1} N$. Then, the following statements are equivalent.

(i) The matrix equations

$$
A X=B, \quad X C=D
$$

have a solution $X \in S R^{n \times n}$. 
(ii) The system of matrix equations

$$
A Y=B, \quad Y C=D, \quad Y A^{\top}=B^{\top}, \quad C^{\top} Y=D^{\top}
$$

have a solution $Y \in R^{n \times n}$; in this case, the symmetric solution of the matrix equations (24) is

$$
X=\frac{Y+Y^{\top}}{2} .
$$

(iii) If

$$
\begin{gathered}
K K^{\dagger} Q_{1} \mathscr{R}_{C}=Q_{1}, \quad Q \mathscr{L}_{N}=0, \quad \mathscr{R}_{\mathscr{L}_{A} \mathscr{L}_{K} Q=0}, \quad A A^{\dagger} B=B, \quad D C^{\dagger} C=D,
\end{gathered}
$$

the symmetric solutions of the matrix equations (24) can be expressed as

$$
\begin{aligned}
X=\frac{1}{2}\left(A^{\dagger} B+\mathscr{L}_{A} D C^{\dagger}+\mathscr{L}_{A} K^{\dagger} Q_{1} \mathscr{R}_{C}\right. \\
\left.+Q^{\dagger} \mathscr{R}_{C}+\mathscr{L}_{A} \mathscr{L}_{K} Z \mathscr{R}_{N} \mathscr{R}_{C}\right) \\
+\frac{1}{2}\left(B^{\top} A^{\top \dagger}+C^{\top \dagger} D^{\top} \mathscr{L}_{A}+\mathscr{R}_{C} Q_{1}^{\top} K^{\top \dagger} \mathscr{L}_{A}\right. \\
\left.+\mathscr{R}_{C} N^{\top \dagger} Q^{\top}+\mathscr{R}_{C} \mathscr{R}_{N} Z^{\top} \mathscr{L}_{K} \mathscr{L}_{A}\right),
\end{aligned}
$$

where $Z \in R^{n \times n}$ is an arbitrary matrix.

Proof. (i) $\Leftrightarrow$ (ii). It is not difficult to get that (i) is equivalent to (ii). Further, if $Y$ is a solution of the matrix equations (25), then

$$
X=\frac{Y+Y^{\top}}{2}=X^{\top} .
$$

Moreover,

$$
\begin{aligned}
& A X=A \frac{Y+Y^{\top}}{2}=\frac{1}{2}\left(A Y+A Y^{\top}\right)=\frac{1}{2}(B+B)=B \\
& X C=\frac{Y+Y^{\top}}{2} C=\frac{1}{2}\left(Y C+Y^{\top} C\right)=\frac{1}{2}(D+D)=D .
\end{aligned}
$$

Then, the expression in (26) is the symmetric solution of the matrix equations (24).

(ii) $\Leftrightarrow$ (iii). From Lemma 11, it can be proved that (ii) is equivalent to (iii) and the solutions of the matrix equations (25) can be expressed as

$$
\begin{aligned}
Y= & A^{\dagger} B+\mathscr{L}_{A} D C^{\dagger}+\mathscr{L}_{A} K^{\dagger} Q_{1} \mathscr{R}_{C} \\
& +Q N^{\dagger} \mathscr{R}_{C}+\mathscr{L}_{A} \mathscr{L}_{K} Z \mathscr{R}_{N} \mathscr{R}_{C} .
\end{aligned}
$$

Substituting (31) into (26) yields (28). The proof is completed.
For $U \in O R^{n \times n}$ which is given by (8), partition

$$
\begin{gathered}
A U=\left(\begin{array}{ll}
A_{1} & A_{2}
\end{array}\right), \quad B U=\left(\begin{array}{ll}
B_{1} & B_{2}
\end{array}\right), \\
A_{1}, B_{1} \in R^{m \times r}, \quad A_{2}, B_{2} \in R^{m \times(n-r)}, \\
C^{\top} U=\left(\begin{array}{ll}
C_{1}^{\top} & C_{2}^{\top}
\end{array}\right), \quad D^{\top} U=\left(\begin{array}{ll}
D_{1}^{\top} & D_{2}^{\top}
\end{array}\right), \\
C_{1}, D_{1} \in R^{r \times l}, \quad C_{2}, D_{2} \in R^{(n-r) \times l} .
\end{gathered}
$$

From Lemma 8, we know that the matrix equations $A X=B$, $X C=D$ have a solution $X \in G B S R^{n \times n}$ if and only if there exist $X_{11} \in S R^{r \times r}$ and $X_{22} \in S R^{(n-r) \times(n-r)}$ such that

$$
\begin{gathered}
A U\left(\begin{array}{cc}
X_{11} & 0 \\
0 & X_{22}
\end{array}\right)=B U, \\
\left(\begin{array}{cc}
X_{11} & 0 \\
0 & X_{22}
\end{array}\right) U^{\top} C=U^{\top} D,
\end{gathered}
$$

that is,

$$
\begin{array}{ll}
A_{1} X_{11}=B_{1}, & X_{11} C_{1}=D_{1}, \\
A_{2} X_{22}=B_{2}, & X_{22} C_{2}=D_{2} .
\end{array}
$$

Let $K_{1}=C_{1}^{\top} \mathscr{L}_{A_{1}}, N_{1}=\mathscr{R}_{C_{1}} A_{1}^{\top}, Q_{1}=D_{1}^{\top}-C_{1}^{\top} A_{1}^{\dagger} B_{1}-$ $K_{1} D_{1} C_{1}^{\dagger}, \bar{Q}=B_{1}^{\top}-A_{1}^{\dagger} B_{1} A_{1}^{\top}-\mathscr{L}_{A_{1}} D_{1} C_{1}^{\dagger} A_{1}^{\top}-\mathscr{L}_{A_{1}} K_{1}^{\dagger} Q_{1} N_{1}$. By Lemma 12, the matrix equations (35) have a solution $X_{11} \in$ $S R^{r \times r}$ if and only if

$$
\begin{aligned}
& K_{1} K_{1}^{\dagger} Q_{1} \mathscr{R}_{C_{1}}=Q_{1}, \quad \bar{Q} \mathscr{L}_{N_{1}}=0, \quad \mathscr{R}_{\mathscr{L}_{A_{1}} \mathscr{L}_{K_{1}}} \bar{Q}=0 \text {, } \\
& A_{1} D_{1}=B_{1} C_{1}, \quad A_{1} A_{1}^{\dagger} B_{1}=B_{1}, \quad D_{1} C_{1}^{\dagger} C_{1}=D_{1} \text {, }
\end{aligned}
$$

in which case the general solutions can be expressed as

$$
\begin{aligned}
X_{11}= & \frac{1}{2}\left(A_{1}^{\dagger} B_{1}+\mathscr{L}_{A_{1}} D_{1} C_{1}^{\dagger}+\mathscr{L}_{A_{1}} K_{1}^{\dagger} Q_{1} \mathscr{R}_{C_{1}}\right. \\
& \left.+\bar{Q} N_{1}^{\dagger} \mathscr{R}_{C_{1}}+\mathscr{L}_{A_{1}} \mathscr{L}_{K_{1}} Z_{1} \mathscr{R}_{N_{1}} \mathscr{R}_{C_{1}}\right) \\
+ & \frac{1}{2}\left(B_{1}^{\top} A_{1}^{\top \dagger}+C_{1}^{\top \dagger} D_{1}^{\top} \mathscr{L}_{A_{1}}+\mathscr{R}_{C_{1}} Q_{1}^{\top} K_{1}^{\top \dagger} \mathscr{L}_{A_{1}}\right. \\
& \left.+\mathscr{R}_{C_{1}} N_{1}^{\top \dagger} \bar{Q}^{\top}+\mathscr{R}_{C_{1}} \mathscr{R}_{N_{1}} Z_{1}^{\top} \mathscr{L}_{K_{1}} \mathscr{L}_{A_{1}}\right)
\end{aligned}
$$

where $Z_{1} \in R^{r \times r}$ is an arbitrary matrix.

Let $K_{2}=C_{2}^{\top} \mathscr{L}_{A_{2}}, N_{2}=\mathscr{R}_{C_{2}} A_{2}^{\top}, Q_{2}=D_{2}^{\top}-C_{2}^{\top} A_{2}^{\dagger} B_{2}-$ $K_{2} D_{2} C_{2}^{\dagger}, \widetilde{Q}=B_{2}^{\top}-A_{2}^{\dagger} B_{2} A_{2}^{\top}-\mathscr{L}_{A_{2}} D_{2} C_{2}^{\dagger} A_{2}^{\top}-\mathscr{L}_{A_{2}} K_{2}^{\dagger} Q_{2} N_{2}$. Similarly, the matrix equations (36) have a solution $X_{22} \in$ $S R^{(n-r) \times(n-r)}$ if and only if

$$
\begin{array}{ccc}
K_{2} K_{2}^{\dagger} Q_{2} \mathscr{R}_{C_{2}}=Q_{2}, & \widetilde{Q} \mathscr{L}_{N_{2}}=0, & \mathscr{R}_{\mathscr{L}_{A_{2}} \mathscr{L}_{K_{2}}} \widetilde{Q}=0, \\
A_{2} D_{2}=B_{2} C_{2}, & A_{2} A_{2}^{\dagger} B_{2}=B_{2}, & D_{2} C_{2}^{\dagger} C_{2}=D_{2},
\end{array}
$$

in which case the general solutions can be expressed as

$$
\begin{aligned}
& X_{22}=\frac{1}{2}\left(A_{2}^{\dagger} B_{2}+\mathscr{L}_{A_{2}} D_{2} C_{2}^{\dagger}+\mathscr{L}_{A_{2}} K_{2}^{\dagger} Q_{2} \mathscr{R}_{C_{2}}\right. \\
&\left.+\widetilde{Q} N_{2}^{\dagger} \mathscr{R}_{C_{2}}+\mathscr{L}_{A_{2}} \mathscr{L}_{K_{2}} Z_{2} \mathscr{R}_{N_{2}} \mathscr{R}_{C_{2}}\right)
\end{aligned}
$$




$$
\begin{gathered}
+\frac{1}{2}\left(B_{2}^{\top} A_{2}^{\top \dagger}+C_{2}^{\top \dagger} D_{2}^{\top} \mathscr{L}_{A_{2}}+\mathscr{R}_{C_{2}} Q_{2}^{\top} K_{2}^{\top \dagger} \mathscr{L}_{A_{2}}\right. \\
\left.+\mathscr{R}_{\mathrm{C}_{2}} N_{2}^{\top \dagger} \widetilde{Q}^{\top}+\mathscr{R}_{\mathrm{C}_{2}} \mathscr{R}_{\mathrm{N}_{2}} Z_{2}^{\top} \mathscr{L}_{K_{2}} \mathscr{L}_{A_{2}}\right)
\end{gathered}
$$

where $Z_{2} \in R^{(n-r) \times(n-r)}$ is an arbitrary matrix.

Now, based on the above discussion, we give the solvability conditions and the general expression of the solutions of Problem 3.

Theorem 13. Given $A, B \in R^{m \times n}, C, D \in R^{n \times l}$. And $U \in$ $O R^{n \times n}$ is given by (8). Let the partitions of $A U, B U, C^{\top} U$, and $D^{\top} U$ be as in (32) and (33), respectively. Then, Problem 3 is consistent if and only if (37) and (39) hold, in which case the general solutions can be expressed as

$$
X=U\left(\begin{array}{cc}
X_{11} & 0 \\
0 & X_{22}
\end{array}\right) U^{\top}
$$

where $X_{11}$ and $X_{22}$ are given as in (38) and (40).

From Lemma 9, we know that the matrix equations $A X=$ $B, X C=D$ have a solution $X \in G B S S R^{n \times n}$ if and only if there exists $X_{12} \in R^{r \times(n-r)}$ such that

$$
\begin{gathered}
A U\left(\begin{array}{cc}
0 & X_{12} \\
-X_{12}^{\top} & 0
\end{array}\right)=B U, \\
\left(\begin{array}{cc}
0 & X_{12} \\
-X_{12}^{\top} & 0
\end{array}\right) U^{\top} C=U^{\top} D,
\end{gathered}
$$

that is,

$$
\begin{array}{ll}
A_{1} X_{12}=B_{2}, & X_{12} A_{2}^{\top}=-B_{1}^{\top}, \\
C_{1}^{\top} X_{12}=-D_{2}^{\top}, & X_{12} C_{2}=D_{1} .
\end{array}
$$

Let $K=C_{1}^{\top} \mathscr{L}_{A_{1}}, N=\mathscr{R}_{A_{2}^{\top}} C_{2}=\mathscr{L}_{A_{2}} C_{2}, Q_{1}=-D_{2}^{\top}-$ $C_{1}^{\top} A_{1}^{\dagger} B_{2}+K B_{1}^{\top} A_{2}^{\top \dagger}, Q=D_{1}-A_{1}^{\dagger} B_{2} C_{2}+\mathscr{L}_{A_{1}} B_{1}^{\top} A_{2}^{\top \dagger} C_{2}-$ $\mathscr{L}_{A_{1}} K^{\dagger} Q_{1} N$. By Lemma 11, the system of matrix equations (43) has a solution $X_{12} \in R^{r \times(n-r)}$ if and only if

$$
\begin{gathered}
K K^{\dagger} Q_{1} \mathscr{R}_{A_{2}^{\top}}=Q_{1}, \quad Q \mathscr{L}_{N}=0, \quad \mathscr{R}_{\mathscr{L}_{A_{1}} \mathscr{L}_{K} Q=0,}, \quad D_{2} C_{1}^{\dagger} C_{1}=D_{2}, \\
A_{1} B_{1}^{\top}=-B_{2} A_{2}^{\top}, \quad A_{1} A_{1}^{\dagger} B_{2}=B_{2}, \quad D_{1} C_{2}^{\dagger} C_{2}=D_{1},
\end{gathered}
$$

in which case the general solutions can be expressed as

$$
\begin{aligned}
X_{12}= & A_{1}^{\dagger} B_{2}-\mathscr{L}_{A_{1}} B_{1}^{\top} A_{2}^{\top \dagger}+\mathscr{L}_{A_{1}} K^{\dagger} Q_{1} \mathscr{R}_{A_{2}^{\top}} \\
& +Q N^{\dagger} \mathscr{R}_{A_{2}^{\top}}+\mathscr{L}_{A_{1}} \mathscr{L}_{K} Z \mathscr{R}_{N} \mathscr{R}_{A_{2}^{\top}},
\end{aligned}
$$

where $Z \in R^{n \times(n-r)}$ is an arbitrary matrix.

Therefore, we have the following result about Problem 4.

Theorem 14. Given $A, B \in R^{m \times n}, C, D \in R^{n \times l}$. And $U \in$ $O R^{n \times n}$ is given by (8). Let the partitions of $A U, B U, C^{\top} U$, and
$D^{\top} U$ be as in (32) and (33), respectively. Then, Problem 4 is consistent if and only if (44) holds, in which case the general solutions can be expressed as

$$
X=U\left(\begin{array}{cc}
0 & X_{12} \\
-X_{12}^{\top} & 0
\end{array}\right) U^{\top}
$$

where $X_{12}$ is given as in (45).

\section{The Generalized Bisymmetric (Bi-Skew- Symmetric) Least Squares Solutions of the Matrix Equations $A X=B, X C=D$}

It is well known that if the solvability conditions of the linear matrix equation or linear matrix equations are not satisfied, we can derive its approximate solutions, among which, the least squares solution is usually considered. In this section, we try to solve the Problems 5 and 6 . Firstly, we present some lemmas which will play important roles in the following.

Lemma 15 (see [14]). Given $A, B \in R^{m \times n}$. Let the singular value decomposition (SVD) of $A$ be

$$
A=W\left(\begin{array}{ll}
\Sigma & 0 \\
0 & 0
\end{array}\right) V^{\top}
$$

where $W=\left(\begin{array}{ll}W_{1} & W_{2}\end{array}\right) \in O R^{m \times m}, V=\left(\begin{array}{ll}V_{1} & V_{2}\end{array}\right) \in O R^{n \times n}$, $\Sigma=\operatorname{diag}\left(\sigma_{1}, \ldots, \sigma_{s}\right)>0, s=\operatorname{rank}(A)$. Then, there exists $\widehat{X} \in$ $S R^{m \times m}$ such that

$$
\|\widehat{X} A-B\|=\min _{X \in S R^{m \times m}}\|X A-B\| .
$$

In this case, $\widehat{X}$ can be expressed as

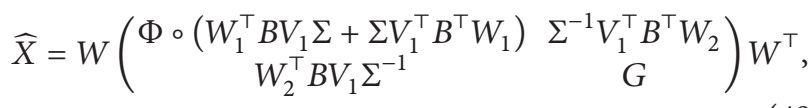

where $\Phi=\left(\varphi_{i j}\right) \in R^{s \times s}, \varphi_{i j}=1 /\left(\sigma_{i}^{2}+\sigma_{j}^{2}\right), 1 \leq i, j \leq s$, and $G \in S R^{(m-s) \times(m-s)}$ is an arbitrary matrix.

Lemma 16 (see [15]). Given $A \in R^{m \times n}, B \in R^{m \times p}, C \in R^{p \times l}$, and $D \in R^{n \times l}$. Let the SVDs of $A$ and $C$ be

$$
A=W\left(\begin{array}{cc}
\Sigma & 0 \\
0 & 0
\end{array}\right) V^{\top}, \quad C=T\left(\begin{array}{cc}
\Omega & 0 \\
0 & 0
\end{array}\right) Q^{\top}
$$

where $W=\left(\begin{array}{ll}W_{1} & W_{2}\end{array}\right), V=\left(\begin{array}{ll}V_{1} & V_{2}\end{array}\right), T=\left(\begin{array}{ll}T_{1} & T_{2}\end{array}\right)$, and $Q=\left(\begin{array}{lll}Q_{1} & Q_{2}\end{array}\right)$ are all orthogonal matrices and the partitions are compatible with the sizes of

$$
\begin{aligned}
\Sigma=\operatorname{diag}\left(\sigma_{1}, \ldots, \sigma_{s}\right)>0, & \Omega=\operatorname{diag}\left(\omega_{1}, \ldots, \omega_{t}\right)>0, \\
s=\operatorname{rank}(A), & t=\operatorname{rank}(C) .
\end{aligned}
$$

Then the least squares solutions of the matrix equations $A X=$ $B, X C=D$ can be expressed as 


$$
\begin{aligned}
& \widehat{X} \\
& =V\left(\begin{array}{cc}
\Phi \circ\left[V_{1}^{\top}\left(A^{\top} B+D C^{\top}\right) T_{1}\right] & \left(\Sigma^{-1}\right)^{2} V_{1}^{\top}\left(A^{\top} B+D C^{\top}\right) T_{2} \\
V_{2}^{\top}\left(A^{\top} B+D C^{\top}\right) T_{1}\left(\Omega^{-1}\right)^{2} & G
\end{array}\right) T^{\top},
\end{aligned}
$$

where $\Phi=\left(\varphi_{i j}\right) \in R^{s \times t}, \varphi_{i j}=1 /\left(\sigma_{i}^{2}+\omega_{j}^{2}\right), 1 \leq i \leq s, 1 \leq j \leq t$, and $G \in R^{(n-s) \times(p-t)}$ is an arbitrary matrix.

$$
\begin{aligned}
& \text { Let the SVDs of }\left(\begin{array}{ll}
A_{1}^{\top} & C_{1}
\end{array}\right) \text { and }\left(\begin{array}{ll}
A_{2}^{\top} & C_{2}
\end{array}\right) \text { be } \\
& \left(\begin{array}{ll}
A_{1}^{\top} & C_{1}
\end{array}\right)=V\left(\begin{array}{ll}
\Sigma & 0 \\
0 & 0
\end{array}\right) W^{\top}, \quad\left(\begin{array}{ll}
A_{2}^{\top} & C_{2}
\end{array}\right)=T\left(\begin{array}{cc}
\Omega & 0 \\
0 & 0
\end{array}\right) Q^{\top},
\end{aligned}
$$

where $V=\left(\begin{array}{ll}V_{1} & V_{2}\end{array}\right), W=\left(\begin{array}{ll}W_{1} & W_{2}\end{array}\right), T=\left(\begin{array}{ll}T_{1} & T_{2}\end{array}\right)$, and $Q=$ $\left(\begin{array}{ll}Q_{1} & Q_{2}\end{array}\right)$ are all orthogonal matrices and the partitions are compatible with the sizes of

$$
\begin{array}{cl}
\Sigma=\operatorname{diag}\left(\sigma_{1}, \ldots, \sigma_{s}\right)>0, & \Omega=\operatorname{diag}\left(\omega_{1}, \ldots, \omega_{t}\right)>0, \\
s=\operatorname{rank}\left(\left(A_{1}^{\top} C_{1}\right)\right), \quad t=\operatorname{rank}\left(\left(A_{2}^{\top} C_{2}\right)\right) .
\end{array}
$$

Based on Lemma 8 and the properties of Frobenius norm, we have

$$
\begin{aligned}
& \|A X-B\|^{2}+\|X C-D\|^{2} \\
& =\left\|A U\left(\begin{array}{cc}
X_{11} & 0 \\
0 & X_{22}
\end{array}\right) U^{\top}-B\right\|^{2} \\
& +\left\|U\left(\begin{array}{cc}
X_{11} & 0 \\
0 & X_{22}
\end{array}\right) U^{\top} C-D\right\|^{2} \\
& =\left\|A_{1} X_{11}-B_{1}\right\|^{2}+\left\|A_{2} X_{22}-B_{2}\right\|^{2} \\
& +\left\|X_{11} C_{1}-D_{1}\right\|^{2}+\left\|X_{22} C_{2}-D_{2}\right\|^{2} \\
& =\left\|X_{11}\left(\begin{array}{ll}
A_{1}^{\top} & C_{1}
\end{array}\right)-\left(\begin{array}{ll}
B_{1}^{\top} & D_{1}
\end{array}\right)\right\|^{2} \\
& +\left\|X_{22}\left(\begin{array}{ll}
A_{2}^{\top} & C_{2}
\end{array}\right)-\left(\begin{array}{ll}
B_{2}^{\top} & D_{2}
\end{array}\right)\right\|^{2} .
\end{aligned}
$$

Hence, $\min _{X \in G B S R^{n \times n}}\|A X-B\|+\|X C-D\|$ is equivalent to

$$
\begin{gathered}
\min _{X_{11} \in S R^{r \times r}}\left\|X_{11}\left(\begin{array}{ll}
A_{1}^{\top} & C_{1}
\end{array}\right)-\left(\begin{array}{ll}
B_{1}^{\top} & D_{1}
\end{array}\right)\right\|, \\
\min _{X_{22} \in S R^{(n-r) \times(n-r)}}\left\|X_{22}\left(\begin{array}{lll}
A_{2}^{\top} & C_{2}
\end{array}\right)-\left(\begin{array}{ll}
B_{2}^{\top} & D_{2}
\end{array}\right)\right\| .
\end{gathered}
$$

From Lemma 15, when $X_{11}$ has the form

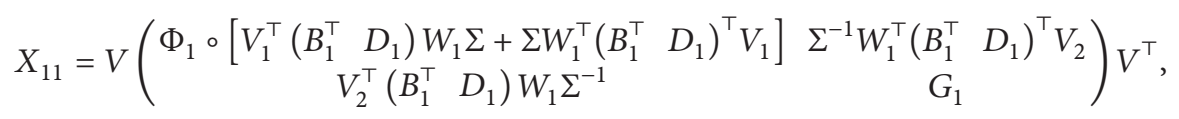

where $\Phi_{1}=\left(\varphi_{i j}\right) \in R^{s \times s}, \varphi_{i j}=1 /\left(\sigma_{i}^{2}+\sigma_{j}^{2}\right), 1 \leq i, j \leq s$, and $G_{1} \in S R^{(r-s) \times(r-s)}$ is an arbitrary matrix, (56) holds.

According to Lemma 15, we know that (57) holds if $X_{22}$ has the form

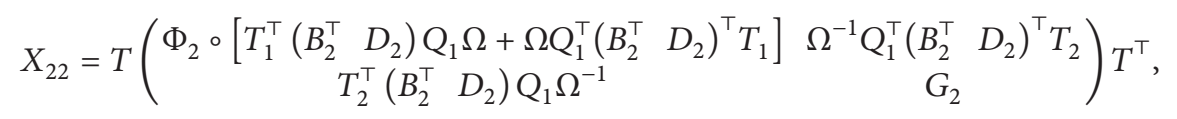

where $\Phi_{2}=\left(\varphi_{i j}\right) \in R^{t \times t}, \varphi_{i j}=1 /\left(\omega_{i}^{2}+\omega_{j}^{2}\right), 1 \leq i, j \leq t$, and $G_{2} \in S R^{(n-r-t) \times(n-r-t)}$ is an arbitrary matrix.

From the above discussion, we get the solutions of Problem 5.

Theorem 17. Given $A, B \in R^{m \times n}, C, D \in R^{n \times l}$. And $U \in$ $O R^{n \times n}$ is given by (8). Let the partitions of $A U, B U, C^{\top} U$, and $D^{\top} U$ be as in (32) and (33), respectively. Let the SVDs of $\left(\begin{array}{lll}A_{1}^{\top} & C_{1}\end{array}\right)$ and $\left(\begin{array}{lll}A_{2}^{\top} & C_{2}\end{array}\right)$ be as (53). Then, the solutions of Problem 5 can be expressed as

$$
X=U\left(\begin{array}{cc}
X_{11} & 0 \\
0 & X_{22}
\end{array}\right) U^{\top},
$$

where $X_{11}$ and $X_{22}$ are given as in (58) and (59).
Now, we give another main result of this section. Based on Lemma 9 and the properties of Frobenius norm, we have

$$
\begin{aligned}
\|A X-B\|^{2}+\|X C-D\|^{2} \\
=\left\|A U\left(\begin{array}{cc}
0 & X_{12} \\
-X_{12}^{\top} & 0
\end{array}\right) U^{\top}-B\right\|^{2} \\
\quad+\left\|U\left(\begin{array}{cc}
0 & X_{12} \\
-X_{12}^{\top} & 0
\end{array}\right) U^{\top} C-D\right\|^{2} \\
=\left\|X_{12} A_{2}^{\top}-\left(-B_{1}^{\top}\right)\right\|^{2}+\left\|A_{1} X_{12}-B_{2}\right\|^{2}
\end{aligned}
$$




$$
\begin{aligned}
& +\left\|X_{12} C_{2}-D_{1}\right\|^{2}+\left\|C_{1}^{\top} X_{12}-\left(-D_{2}^{\top}\right)\right\|^{2} \\
= & \left\|\left(\begin{array}{c}
A_{1} \\
C_{1}^{\top}
\end{array}\right) X_{12}-\left(\begin{array}{c}
B_{2} \\
-D_{2}^{\top}
\end{array}\right)\right\|^{2} \\
& +\left\|X_{12}\left(\begin{array}{ll}
A_{2}^{\top} & C_{2}
\end{array}\right)-\left(\begin{array}{ll}
-B_{1}^{\top} & D_{1}
\end{array}\right)\right\|^{2} .
\end{aligned}
$$

Then, from Lemma 16, the least squares solutions of the matrix equations

$$
\left(\begin{array}{l}
A_{1} \\
C_{1}^{\top}
\end{array}\right) X_{12}=\left(\begin{array}{c}
B_{2} \\
-D_{2}^{\top}
\end{array}\right), \quad X_{12}\left(\begin{array}{ll}
A_{2}^{\top} & C_{2}
\end{array}\right)=\left(\begin{array}{ll}
-B_{1}^{\top} & D_{1}
\end{array}\right)
$$

can be written as

$$
X_{12}=V\left(\begin{array}{cc}
\Phi \circ\left(V_{1}^{\top} X_{0} T_{1}\right) & \left(\Sigma^{-1}\right)^{2} V_{1}^{\top} X_{0} T_{2} \\
V_{2}^{\top} X_{0} T_{1}\left(\Omega^{-1}\right)^{2} & G
\end{array}\right) T^{\top}
$$

where $X_{0}=A_{1}^{\top} B_{2}-B_{1}^{\top} A_{2}-C_{1} D_{2}^{\top}+D_{1} C_{2}^{\top}, \Phi=\left(\varphi_{i j}\right) \in R^{s \times t}$, $\varphi_{i j}=1 /\left(\sigma_{i}^{2}+\omega_{j}^{2}\right), 1 \leq i \leq s, 1 \leq j \leq t$, and $G \in R^{(r-s) \times(n-r-t)}$ is an arbitrary matrix.

Theorem 18. Given $A, B \in R^{m \times n}, C, D \in R^{n \times l}$. And $U \in$ $O R^{n \times n}$ is given by (8). Let the partitions of $A U, B U, C^{\top} U$, and $D^{\top} U$ be as in (32) and (33), respectively. Let the SVDs of $\left(\begin{array}{lll}A_{1}^{\top} & C_{1}\end{array}\right)$ and $\left(\begin{array}{ll}A_{2}^{\top} & C_{2}\end{array}\right)$ be as (53). Then, the solutions of Problem 6 can be expressed as

$$
X=U\left(\begin{array}{cc}
0 & X_{12} \\
-X_{12}^{\top} & 0
\end{array}\right) U^{\top}
$$

where $X_{12}$ is given as in (63).

\section{Numerical Examples}

In this section, we provide two algorithms to compute the generalized bisymmetric (bi-skew-symmetric) solution and the generalized bisymmetric (bi-skew-symmetric) least squares solution of the matrix equations $A X=B, X C=D$ and give some examples to illustrate the efficiency of our proposed algorithms.

Algorithm 1 (the algorithm about Problems 3 and 5).

Step 1. Input $A, B, C, D, P$.

Step 2. Compute $A_{1}, A_{2}, B_{1}, B_{2}, C_{1}, C_{2}, D_{1}, D_{2}$ by (32) and (33).

Step 3. If any of conditions in (37) and (39) does not hold, then turn to Step 4. Otherwise, compute the generalized bisymmetric solution of the matrix equations $A X=B, X C=$ $D$ by Theorem 13 .

Step 4. Compute the generalized bisymmetric least squares solution of the matrix equations $A X=B, X C=D$ by Theorem 17.
Algorithm 2 (the algorithm about Problems 4 and 6).

Step 1. Input $A, B, C, D, P$.

Step 2. Compute $A_{1}, A_{2}, B_{1}, B_{2}, C_{1}, C_{2}, D_{1}, D_{2}$ by (32) and (33).

Step 3. If any of conditions in (44) does not hold, then turn to Step 4. Otherwise, compute the generalized bi-skewsymmetric solution of the matrix equations $A X=B, X C=D$ by Theorem 14 .

Step 4. Compute the generalized bi-skew-symmetric least squares solution of the matrix equations $A X=B, X C=D$ by Theorem 18.

Example 1. Given $A, B, C, D \in R^{3 \times 3}, P \in S O R^{3 \times 3}$ as follows:

$$
\begin{aligned}
A & =\left(\begin{array}{lll}
0.9649 & 0.9572 & 0.1419 \\
0.1576 & 0.4854 & 0.4218 \\
0.9706 & 0.8003 & 0.9157
\end{array}\right) \\
B & =\left(\begin{array}{ccc}
2.6396 & 2.2226 & -0.8424 \\
0.2559 & 0.9047 & 0.1875 \\
2.4422 & 1.2061 & 0.4122
\end{array}\right) \\
C & =\left(\begin{array}{ccc}
0.7922 & 0.0357 & 0.6787 \\
0.9595 & 0.8491 & 0.7577 \\
0.6557 & 0.9340 & 0.7431
\end{array}\right) \\
D & =\left(\begin{array}{ccc}
1.9731 & -0.3179 & 1.6446 \\
1.8522 & 1.4664 & 1.2772 \\
-0.0385 & 0.6894 & 0.2831
\end{array}\right) \\
P & =\left(\begin{array}{ccc}
0.2140 & 0.9246 & -0.3151 \\
0.9246 & -0.0878 & 0.3707 \\
-0.3151 & 0.3707 & 0.8737
\end{array}\right)
\end{aligned}
$$

Then, there exists

$$
U=\left(\begin{array}{ccc}
-0.6612 & -0.4121 & -0.6269 \\
-0.6742 & -0.0400 & 0.7375 \\
-0.3290 & 0.9103 & -0.2513
\end{array}\right) \in O R^{3 \times 3}
$$

such that

$$
P=U\left(\begin{array}{ccc}
1 & 0 & 0 \\
0 & 1 & 0 \\
0 & 0 & -1
\end{array}\right) U^{\top}
$$

(1) By Algorithm 1, the conditions in (37) and (39) hold. Hence, the matrix equations $A X=B, X C=D$ have a generalized bisymmetric solution

$$
X_{G B S}^{*} \approx\left(\begin{array}{ccc}
2.9380 & -0.1583 & -0.3089 \\
-0.1583 & 2.5979 & -0.7858 \\
-0.3089 & -0.7858 & 1.4643
\end{array}\right)
$$


Furthermore,

$$
\begin{gathered}
\left\|A X_{G B S}^{*}-B\right\|=2.2775 e-004, \\
\left\|X_{G B S}^{*} C-D\right\|=2.2231 e-004, \\
\left\|X_{G B S}^{*}-X_{G B S}^{* \top}\right\|=3.5108 e-016, \\
\left\|X_{G B S}^{*}-P X_{G B S}^{*} P\right\|=7.6271 e-004 .
\end{gathered}
$$

(2) By Algorithm 2, at least one of conditions in (44) does not hold. Hence, we get the generalized bi-skew-symmetric least squares solution of the matrix equations $A X=B$, $X C=D$, and the generalized bi-skew-symmetric least squares solution is

$$
X_{\text {GBSS }}=\left(\begin{array}{ccc}
0 & 0.2668 & -0.4629 \\
-0.2668 & 0 & 0.4377 \\
0.4629 & -0.4377 & 0
\end{array}\right)
$$

Furthermore,

$$
\begin{gathered}
\min _{X \in G B S S R^{n \times n}}\|A X-B\|+\|X C-D\|=8.3276, \\
\left\|X_{G B S S}+X_{G B S S}^{\top}\right\|=1.2413 e-016, \\
\left\|X_{G B S S}+P X_{G B S S} P\right\|=1.4017 e-004 .
\end{gathered}
$$

Example 2. Given $A, B, C, D \in R^{4 \times 4}, P \in S O R^{4 \times 4}$ as follows:

$$
\begin{aligned}
A & =\left(\begin{array}{cccc}
0.8147 & 0.6324 & 0.9575 & 0.9572 \\
0.9058 & 0.0975 & 0.9649 & 0.4854 \\
0.1270 & 0.2785 & 0.1576 & 0.8003 \\
0.9134 & 0.5469 & 0.9706 & 0.1419
\end{array}\right) \\
B & =\left(\begin{array}{cccc}
0.5972 & 0.8079 & 0.2988 & -1.3409 \\
-0.0105 & 0.5625 & 0.5447 & -1.1762 \\
0.6749 & 0.4230 & 0.1967 & -0.2930 \\
-0.0796 & 0.4102 & 0.0463 & -1.3852
\end{array}\right) \\
C & =\left(\begin{array}{ccccc}
0.2769 & 0.6948 & 0.4387 & 0.1869 \\
0.0462 & 0.3171 & 0.3816 & 0.4898 \\
0.0971 & 0.9502 & 0.7655 & 0.4456 \\
0.8235 & 0.0344 & 0.7952 & 0.6463
\end{array}\right) \\
D & =\left(\begin{array}{cccc}
-0.6165 & 0.2563 & -0.4455 & -0.5208 \\
-0.3196 & -0.4437 & -0.7539 & -0.5512 \\
-0.4543 & -0.0793 & -0.2536 & 0.0091 \\
0.2788 & 1.1039 & 0.8516 & 0.5620
\end{array}\right) \\
P & =\left(\begin{array}{cccc}
-0.0913 & -0.3887 & 0.8868 & 0.2326 \\
-0.3887 & 0.5024 & -0.0224 & 0.7720 \\
0.8868 & -0.0224 & -0.0391 & 0.4598 \\
0.2326 & 0.7720 & 0.4598 & -0.3721
\end{array}\right)
\end{aligned}
$$

Then, there exists

$$
U=\left(\begin{array}{cccc}
-0.4583 & 0.4943 & -0.3030 & -0.6737 \\
-0.4033 & -0.7672 & -0.4944 & -0.0661 \\
-0.6074 & 0.3339 & -0.1177 & 0.7111 \\
-0.5082 & -0.2359 & 0.8062 & -0.1899
\end{array}\right) \in O R^{4 \times 4}
$$

such that

$$
P=U\left(\begin{array}{cccc}
1 & 0 & 0 & 0 \\
0 & 1 & 0 & 0 \\
0 & 0 & -1 & 0 \\
0 & 0 & 0 & -1
\end{array}\right) U^{\top}
$$

(1) By Algorithm 1, at least one of conditions in (37) and (39) does not hold. Hence, we get the generalized bisymmetric least squares solution of the matrix equations $A X=B, X C=D$ and the generalized bisymmetric least squares solution is

$$
X_{G B S}=\left(\begin{array}{cccc}
0.8056 & 0.3706 & -0.5480 & -0.3864 \\
0.3706 & 0.2007 & -0.2672 & -0.2756 \\
-0.5480 & -0.2672 & 0.3995 & 0.1788 \\
-0.3864 & -0.2756 & 0.1788 & 0.2760
\end{array}\right)
$$

Furthermore,

$$
\begin{gathered}
\min _{X \in G B S R^{n \times n}}\|A X-B\|+\|X C-D\|=4.7963, \\
\left\|X_{G B S}-X_{G B S}^{\top}\right\|=1.7988 e-016, \\
\left\|X_{G B S}-P X_{G B S} P\right\|=1.4279 e-004 .
\end{gathered}
$$

(2) By Algorithm 2, the conditions in (44) hold. Hence, the matrix equations $A X=B, X C=D$ have a generalized bi-skew-symmetric solution

$$
X_{\text {GBSS }}^{*} \approx\left(\begin{array}{cccc}
0 & -0.4451 & 0.4464 & -0.7763 \\
0.4451 & 0 & -0.7764 & -0.4461 \\
-0.4464 & 0.7764 & 0 & -0.4451 \\
0.7763 & 0.4461 & 0.4451 & 0
\end{array}\right)
$$

Furthermore,

$$
\begin{aligned}
\left\|A X_{G B S S}^{*}-B\right\| & =2.6885 e-004, \\
\left\|X_{G B S S}^{*} C-D\right\| & =2.5515 e-004, \\
\left\|X_{G B S S}^{*}+X_{G B S S}^{* T}\right\| & =4.4948 e-016, \\
\left\|X_{G B S S}^{*}+P X_{G B S S}^{*} P\right\| & =1.5075 e-004 .
\end{aligned}
$$


Example 3. Given $A, B \in R^{6 \times 5}, C, D \in R^{5 \times 4}$, and $P \in S O R^{5 \times 5}$ as follows:

$$
\begin{aligned}
& A=\left(\begin{array}{lllll}
0.8147 & 0.2785 & 0.9572 & 0.7922 & 0.6787 \\
0.9058 & 0.5469 & 0.4854 & 0.9595 & 0.7577 \\
0.1270 & 0.9575 & 0.8003 & 0.6557 & 0.7431 \\
0.9134 & 0.9649 & 0.1419 & 0.0357 & 0.3922 \\
0.6324 & 0.1576 & 0.4218 & 0.8491 & 0.6555 \\
0.0975 & 0.9706 & 0.9157 & 0.9340 & 0.1712
\end{array}\right) \\
& B=\left(\begin{array}{lllll}
0.7060 & 0.6948 & 0.7655 & 0.7094 & 0.1190 \\
0.0318 & 0.3171 & 0.7952 & 0.7547 & 0.4984 \\
0.2769 & 0.9502 & 0.1869 & 0.2760 & 0.9597 \\
0.0462 & 0.0344 & 0.4898 & 0.6797 & 0.3404 \\
0.0971 & 0.4387 & 0.4456 & 0.6551 & 0.5853 \\
0.8235 & 0.3816 & 0.6463 & 0.1626 & 0.2238
\end{array}\right), \\
& C=\left(\begin{array}{llll}
0.7513 & 0.9593 & 0.8407 & 0.3500 \\
0.2551 & 0.5472 & 0.2543 & 0.1966 \\
0.5060 & 0.1386 & 0.8143 & 0.2511 \\
0.6991 & 0.1493 & 0.2435 & 0.6160 \\
0.8909 & 0.2575 & 0.9293 & 0.4733
\end{array}\right) \\
& D=\left(\begin{array}{llll}
0.3517 & 0.2858 & 0.0759 & 0.1299 \\
0.8308 & 0.7572 & 0.0540 & 0.5688 \\
0.5853 & 0.7537 & 0.5308 & 0.4694 \\
0.5497 & 0.3804 & 0.7792 & 0.0119 \\
0.9172 & 0.5678 & 0.9340 & 0.3371
\end{array}\right) \\
& P=\left(\begin{array}{ccccc}
-0.1397 & -0.2600 & 0.9091 & 0.2239 & 0.1904 \\
-0.2600 & 0.6869 & 0.0500 & -0.1319 & 0.6638 \\
0.9091 & 0.0500 & 0.1771 & -0.2921 & 0.2332 \\
0.2239 & -0.1319 & -0.2921 & 0.8240 & 0.4098 \\
0.1904 & 0.6638 & 0.2332 & 0.4098 & -0.5483
\end{array}\right)
\end{aligned}
$$

Then, there exists

$$
\begin{aligned}
U & =\left(\begin{array}{ccccc}
-0.3882 & 0.4871 & 0.2054 & -0.0715 & -0.7515 \\
-0.4756 & -0.3492 & -0.7038 & -0.3709 & -0.1377 \\
-0.4034 & 0.6471 & -0.0840 & -0.1630 & 0.6204 \\
-0.5101 & -0.4573 & 0.6653 & -0.2416 & 0.1720 \\
-0.4474 & -0.1134 & -0.1130 & 0.8788 & 0.0430
\end{array}\right) \\
& \in O R^{4 \times 4}
\end{aligned}
$$

such that

$$
P=U\left(\begin{array}{ccccc}
1 & 0 & 0 & 0 & 0 \\
0 & 1 & 0 & 0 & 0 \\
0 & 0 & 1 & 0 & 0 \\
0 & 0 & 0 & -1 & 0 \\
0 & 0 & 0 & 0 & -1
\end{array}\right) U^{\top}
$$

(1) By Algorithm 1, it is verified that any of conditions in (37) and (39) does not hold. Hence, we get the generalized bisymmetric least squares solution of the matrix equations
$A X=B, X C=D$, and the generalized bisymmetric least squares solution is

$$
X_{G B S}=\left(\begin{array}{ccccc}
-0.1363 & 0.1110 & 0.3857 & 0.3289 & 0.0153 \\
0.1110 & 0.3641 & 0.1631 & 0.2608 & 0.0069 \\
0.3857 & 0.1631 & -0.1462 & 0.2554 & 0.1547 \\
0.3289 & 0.2608 & 0.2554 & -0.0403 & 0.0532 \\
0.0153 & 0.0069 & 0.1547 & 0.0532 & 0.6063
\end{array}\right) .
$$

Furthermore,

$$
\begin{gathered}
\min _{X \in G B S R^{n \times n}}\|A X-B\|+\|X C-D\|=2.3819, \\
\left\|X_{G B S}-X_{G B S}^{\top}\right\|=1.9192 e-016 \\
\left\|X_{G B S}-P X_{G B S} P\right\|=1.9312 e-004 .
\end{gathered}
$$

(2) By Algorithm 2, it is verified that any of conditions in (44) does not hold. Hence, we get the generalized bi-skewsymmetric least squares solution of the matrix equations $A X=B, X C=D$, and the generalized bi-skew-symmetric least squares solution is

$$
\begin{aligned}
& X_{\text {GBSS }} \\
& =\left(\begin{array}{ccccc}
0 & -0.0977 & -0.1843 & 0.2895 & -0.1616 \\
0.0977 & 0 & -0.2626 & -0.0055 & 0.1672 \\
0.1843 & 0.2626 & 0 & -0.1968 & -0.1138 \\
-0.2895 & 0.0055 & 0.1968 & 0 & 0.1110 \\
0.1616 & -0.1672 & 0.1138 & -0.1110 & 0
\end{array}\right) .
\end{aligned}
$$

Furthermore,

$$
\begin{gathered}
\min _{X \in G B S S R^{n \times n}}\|A X-B\|+\|X C-D\|=5.3861, \\
\left\|X_{G B S S}+X_{G B S S}^{\top}\right\|=1.2889 e-016, \\
\left\|X_{G B S S}+P X_{G B S S} P\right\|=1.4358 e-004 .
\end{gathered}
$$

\section{Conclusions}

This paper is devoted to considering the generalized bisymmetric (bi-skew-symmetric) solutions of matrix equations $A X=B, X C=D$, and the necessary and sufficient conditions for the solvability and the general expression of the solutions are obtained. If the solvability conditions are not satisfied, the generalized bisymmetric (bi-skewsymmetric) least squares solution of the matrix equations is considered. As an auxiliary, two algorithms have been provided to compute the generalized bisymmetric (bi-skewsymmetric) solutions and the generalized bisymmetric (biskew-symmetric) least squares solution, and some examples have been given to illustrate that the results are reasonable.

\section{Conflict of Interests}

The authors declare that there is no conflict of interests regarding the publication of this paper. 


\section{Authors' Contributions}

All authors contributed equally and significantly in writing this paper. All authors read and approved the final paper.

\section{Acknowledgments}

The project is supported by the National Natural Science Foundation of China (11071041, 11201074), Fujian Natural Science Foundation (2013J01006), and the Scientific Research Special Fund Project of Fujian University (JK2013060). t

\section{References}

[1] F. Cecioni, "Sopra alcune operazioni algebriche sulle matrici," Annali della Scuola Normale Superiore di Pisa, vol. 11, pp. 17-20, 1910.

[2] C. R. Rao and S. K. Mitra, Generalized Inverse of Matrices and Its Applications, John Wiley \& Sons, New York, NY, USA, 1971.

[3] S. K. Mitra, "The matrix equations $A X=C, X B=D$," Linear Algebra and its Applications, vol. 59, pp. 171-181, 1984.

[4] K.-W. E. Chu, "Singular value and generalized singular value decompositions and the solution of linear matrix equations," Linear Algebra and its Applications, vol. 88-89, pp. 83-98, 1987.

[5] Q.-W. Wang and J. Yu, "Constrained solutions of a system of matrix equations," Journal of Applied Mathematics, vol. 2012, Article ID 471573, 19 pages, 2012.

[6] C. G. Khatri and S. K. Mitra, "Hermitian and nonnegative definite solutions of linear matrix equations," SIAM Journal on Applied Mathematics, vol. 31, no. 4, pp. 579-585, 1976.

[7] A. Dajić and J. J. Koliha, "Positive solutions to the equations $A X=C$ and $X B=D$ for Hilbert space operators," Journal of Mathematical Analysis and Applications, vol. 333, no. 2, pp. 567-576, 2007.

[8] F.-L. Li, X.-Y. Hu, and L. Zhang, "The generalized reflexive solution for a class of matrix equations $(A X=B, X C=D)$," Acta Mathematica Scientia, vol. 28, no. 1, pp. 185-193, 2008.

[9] F.-L. Li, X.-Y. Hu, and L. Zhang, "The generalized anti-reflexive solutions for a class of matrix equations $(B X=C, X D=E)$," Computational \& Applied Mathematics, vol. 27, no. 1, pp. 31-46, 2008.

[10] Y.-Y. Qiu, Z.-Y. Zhang, and J. -F. Lu, "The matrix equations $A X=B, X C=D$ with $P X=s X P$ constraint," Applied Mathematics and Computation, vol. 189, no. 2, pp. 1428-1434, 2007.

[11] Q.-W. Wang and J. Yu, "On the generalized bi (skew-) symmetric solutions of a linear matrix equation and its procrust problems," Applied Mathematics and Computation, vol. 219, no. 19, pp. 9872-9884, 2013.

[12] Q.-F. Xiao, X.-Y. Hu, and L. Zhang, "The anti-symmetric orthosymmetric solutions of the matrix equation $A^{T} X A=D$," Electronic Journal of Linear Algebra, vol. 18, pp. 21-29, 2009.

[13] Q.-W. Wang, "A system of four matrix equations over von Neumann regular rings and its applications," Acta Mathematica Sinica, vol. 21, no. 2, pp. 323-334, 2005.

[14] J.-G. Sun, "Two kinds of inverse eigenvalue problems for real symmetric matrices," Mathematica Numerica Sinica, vol. 10, no. 3, pp. 282-290, 1988.

[15] Y.-X. Yuan, "Least-squares solutions to the matrix equations $A X=B$ and $X C=D$," Applied Mathematics and Computation, vol. 216, no. 10, pp. 3120-3125, 2010.
[16] Q.-W. Wang, X. Liu, and S.-W. Yu, “The common bisymmetric nonnegative definite solutions with extreme ranks and inertias to a pair of matrix equations," Applied Mathematics and Computation, vol. 218, no. 6, pp. 2761-2771, 2011.

[17] C.-Z. Dong, Q.-W. Wang, and Y.-P. Zhang, "On the Hermitian $R$-conjugate solution of a system of matrix equations," Journal of Applied Mathematics, vol. 2012, Article ID 398085, 14 pages, 2012.

[18] Q. Zhang and Q.-W. Wang, "The $(P, Q)$-(skew)symmetric extremal rank solutions to a system of quaternion matrix equations," Applied Mathematics and Computation, vol. 217, no. 22, pp. 9286-9296, 2011.

[19] H.-X. Chang, Q.-W. Wang, and G.-J. Song, “( $R, S)$-conjugate solution to a pair of linear matrix equations," Applied Mathematics and Computation, vol. 217, no. 1, pp. 73-82, 2010.

[20] Q.-W. Wang, "The general solution to a system of real quaternion matrix equations," Computers \& Mathematics with Applications, vol. 49, no. 5-6, pp. 665-675, 2005.

[21] Q.-W. Wang, "Bisymmetric and centrosymmetric solutions to systems of real quaternion matrix equations," Computers \& Mathematics with Applications, vol. 49, no. 5-6, pp. 641-650, 2005.

[22] Q.-W. Wang, F. Qin, and C.-Y. Lin, "The common solution to matrix equations over a regular ring with applications," Indian Journal of Pure and Applied Mathematics, vol. 36, no. 12, pp. 655$672,2005$.

[23] Q.-W. Wang and G.-H. Yu, "The least-square bisymmetric solution to a quaternion matrix equation with applications," Bulletin of Iranian Mathematical Society, vol. 39, no. 2, pp. 239257, 2013

[24] Q.-W. Wang, Y. Zhou, and Q. Zhang, "Ranks of the common solution to six quaternion matrix equations," Acta Mathematicae Applicatae Sinica, vol. 27, no. 3, pp. 443-462, 2011.

[25] Q.-W. Wang, J. W. van der Woude, and H.-X. Chang, "A system of real quaternion matrix equations with applications," Linear Algebra and its Applications, vol. 431, no. 12, pp. 2291-2303, 2009.

[26] Q.-W. Wang and F. Zhang, "The reflexive re-nonnegative definite solution to a quaternion matrix equation," Electronic Journal of Linear Algebra, vol. 17, pp. 88-101, 2008.

[27] Q.-W. Wang, G.-J. Song, and C.-Y. Lin, "Rank equalities related to the generalized inverse $A_{T, S}^{(2)}$ with applications," Applied Mathematics and Computation, vol. 205, no. 1, pp. 370-382, 2008.

[28] Q.-W. Wang, H.-X. Chang, and Q. Ning, "The common solution to six quaternion matrix equations with applications," Applied Mathematics and Computation, vol. 198, no. 1, pp. 209-226, 2008.

[29] Q.-W. Wang, H.-X. Chang, and C.-Y. Lin, " $P$-(skew)symmetric common solutions to a pair of quaternion matrix equations," Applied Mathematics and Computation, vol. 195, no. 2, pp. 721732, 2008.

[30] Q.-W. Wang, S.-W. Yu, and C.-Y. Lin, "Extreme ranks of a linear quaternion matrix expression subject to triple quaternion matrix equations with applications," Applied Mathematics and Computation, vol. 195, no. 2, pp. 733-744, 2008.

[31] J. Yu, Q.-W. Wang, and C.-Z. Dong, "(Anti-)Hamiltonian solution to a system of matrix equations," Mathematical Problems in Engineering, vol. 2014, Article ID 539215, 13 pages, 2014. 


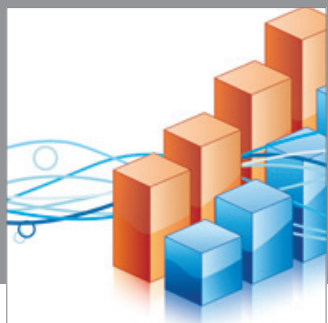

Advances in

Operations Research

mansans

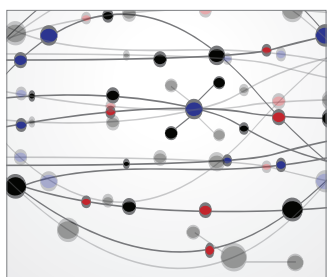

The Scientific World Journal
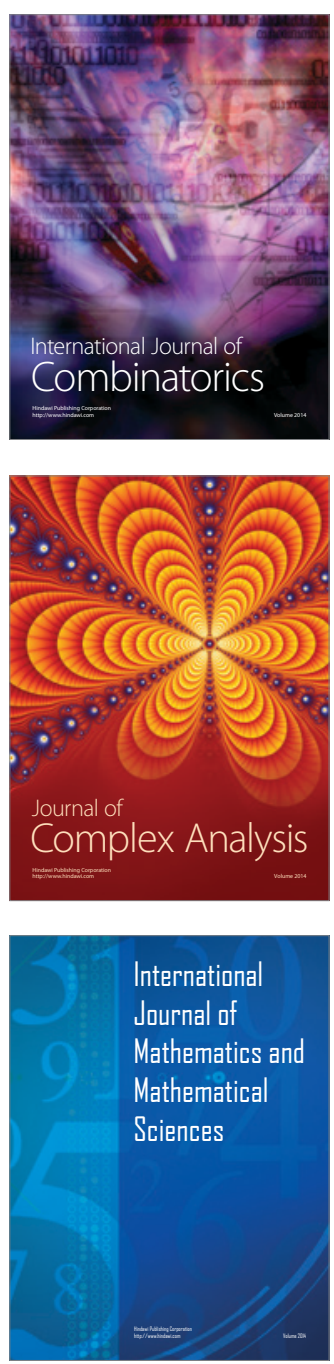
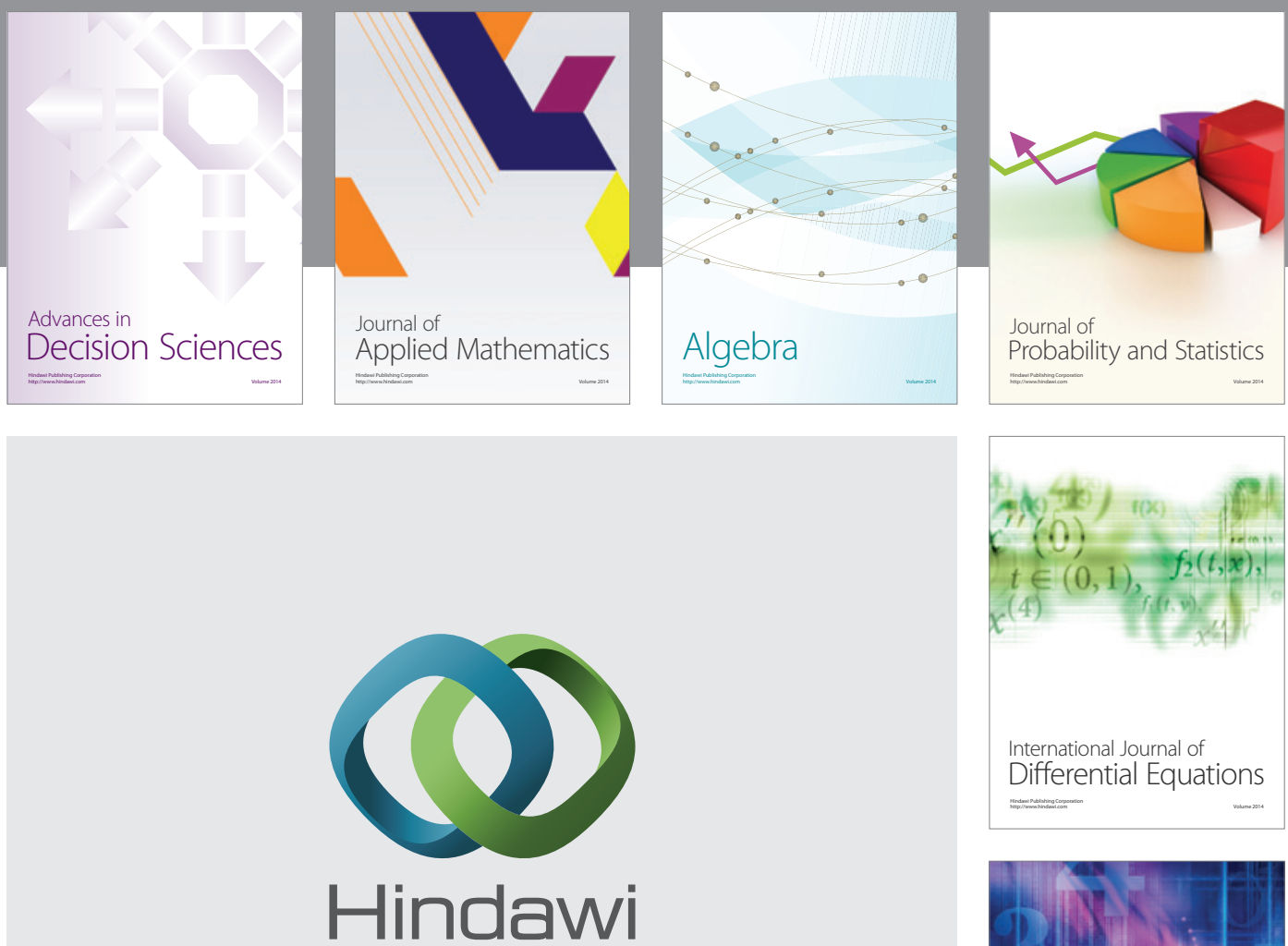

Submit your manuscripts at http://www.hindawi.com
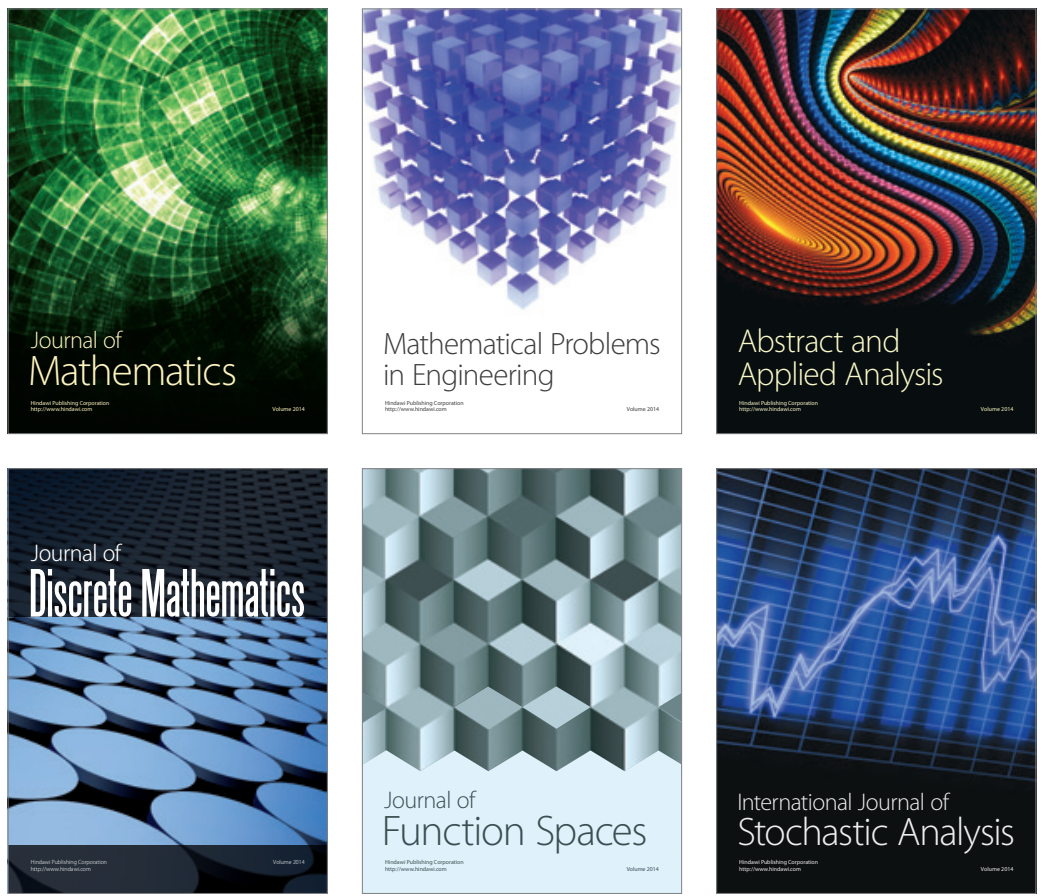

Journal of

Function Spaces

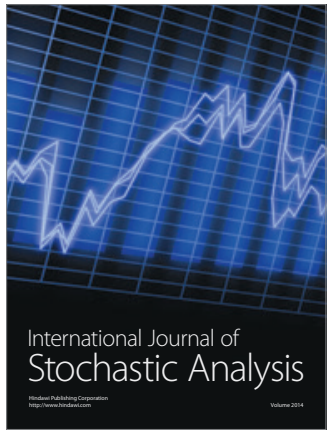

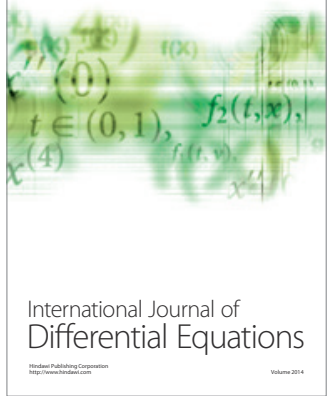
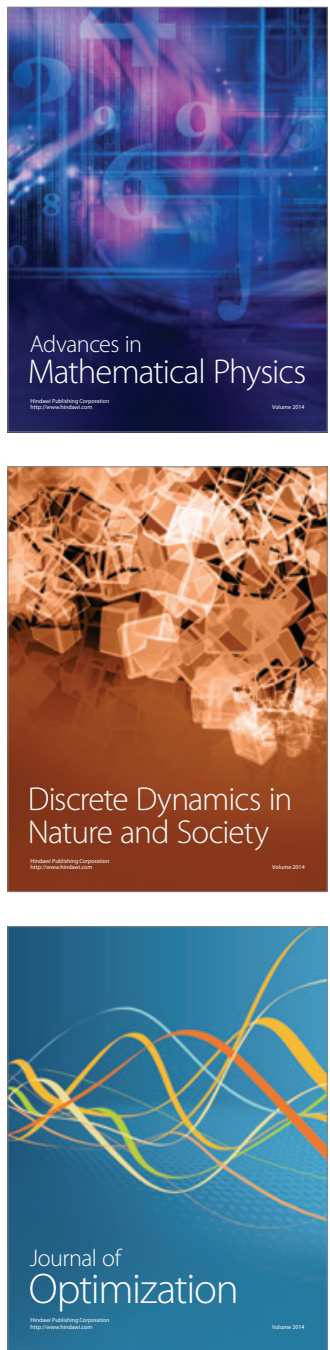